\title{
Prevalence of Soil Transmitted Helminths and Associated Risk Factors among Children Residents in Bandjoun, West Region of Cameroon
}

\author{
Dzune Fossouo Dirane Cleopas ${ }^{1,2 *}$ and Yondo Jeannette ${ }^{3,4}$ \\ ${ }^{1}$ Center for Research on Filariasis and others Tropical Diseases, Cameroon \\ ${ }^{2}$ Department of Biochemistry, Faculty of Medicine and Biomedical Sciences, University \\ of Yaounde 1, Cameroon \\ ${ }^{3}$ Department of Biology Sciences, Faculty of Medicine and Pharmaceutic Sciences, \\ University of Dschang, Cameroon \\ ${ }^{4}$ Department of Animal Biology, University of Dschang, Cameroon \\ *Corresponding Author: Dzune Fossouo Dirane Cleopas, Center for Research on \\ Filariasis and others Tropical Diseases, Cameroon.
}

\author{
Received: August 19, 2020 \\ Published: October 07, 2020 \\ (C) All rights are reserved by Dzune Fossouo \\ Dirane Cleopas and Yondo Jeannette.
}

\begin{abstract}
Geohelminth infections, such as Ascaris lumbricoides, Trichuris trichiura and Hookworms are major public health concerns. These helminths distributed throughout the world with high prevalence in poor and socio-economically deprived communities in the tropics and subtropics cause morbidity and sometimes death. Our study aimed at evaluating the prevalence and intensity of infection of geohelminths and the risk factor in Bandjoun, West Region of Cameroon. Qualitative and quantitative analyses were carried out on three hundred and fifteen (315) stool samples collected from residents using the simple centrifugal flotation and McMaster count technique respectively. Out of the 315 samples examined, twenty-one (6.7\%) were infected with the eggs of at least one helminth parasite with prevalence and intensities of infection of $6.7 \%$ and $6971.4286 \pm 14662.4228$ for Ascaris lumbricoides and $0.3 \%$ and 50 \pm 00 for Trichuris trichiura. More males (11 children) than females (10 children) were infected. The prevalence of $A$. lumbricoides and T. trichiura varied significantly with age groups with those of 1- 10 years taking the lead (12.97\%) and 11-15 years (7.14\%). One double infection (1\%) was recorded with A. lumbricoides + T. trichiura. According to fecal concentration of eggs, $22.72 \%$ of infections were light, $13.6 \%$ moderate and $63.63 \%$ heavy. Factors such as poor hygiene and life conditions, lack of drinking water and lack of knowledge on how to fights the geohelminths were found to be important risk factors for geohelminths. This work shows a relatively low prevalence of geohelminths in this area, proof that the national deworming campaigns are achieving success.
\end{abstract}

Keywords: Geohelminths; Prevalence; Children Residents; Bandjoun; Risk Factor

\section{Abbreviations}

STHs: Soil-Transmitted Helminths; WHO: World Health Organization; FMPS: Faculty of Medicine and Pharmaceutical Sciences; FMBS: Faculty of Medicine and Biomedical Sciences

\section{Introduction}

Geohelminths or soil-transmitted helminths (STHs) are a group of intestinal nematodes composed of A. lumbricoïdes (round- worm), T. trichiura (whipworm), Necator americanus and Ancylostoma duodenale (hookworms). In the majority of low-income countries, poverty, socio-economic status, lack of clean water and precary hygiene favour the development of soil parasites [1]. The World Health Organization (WHO) estimates over 2 billion people in the world living with these parasitic infections are school age children and women of childbearing age [2]. These helminthiases are responsible for anemia, abdominal pain, stunted growth among 
children and pregnant women. Some studies released in the world revealed the prevalence of gastro-intestinal helminths. In Philippines and Mexico, 88\% and 35.2 million of inhabitants are respectively infected by these parasites [3]. In 2004, a report from the Cameroonian Public health Secretary of state revealed 16.1 million of inhabitants in Cameroon with more than 10 million are infected with helminthiasis [8]. Given that there is limited information in Bandjoun regarding the occurrence of soil-transmitted helminths (STHs), our main objective was to determine the prevalence and intensity of infection of geohelminthiasis and associated risk factors among residents.

\section{Materials and Methods}

\section{Study area}

This study was carried out in Bandjoun situated along the West Region of Cameroon, Department of Koung-Khi with three Divisions: Poumougne, Djebem and Bayangam. Bandjoun has about 6872 residents. It is located between Latitude $5^{\circ} 22^{\prime} 32^{\prime \prime}$ North and Longitude $10^{\circ} 24^{\prime} 47^{\prime \prime}$ East. It is limited in the North by Bafoussam; in the South by Bagang Fokam; in the East by Foumban and West by Baham, Bahouan. This city has a cool, mild climate (wet tropical) of the Equatorial Guinean type characterized by two seasons: a rainy season that runs from March to November and a dry season from November to March.

\section{Study population}

Our target population consisted of children aged 1 to 15 living in the Kamgo, Ha'a and Tesse quarters of Bandjoun and those referred to the laboratory of the Mbouo hospital for a stool examination. These quarters were selected in relation to their problems of access to drinking water. So, three hundred and fifteen (315) subjects were randomly selected between April and July 2017. Of these children, 64 were non-school age children; 214 and 39 children were in primary and secondary school respectively. The study population was made up of 149 (47.30\%) females and 166 $(52.70 \%)$ males.

\section{Parasitological examination and risk factor determination}

Coprological analysis was done to have a qualitative and quantitative appreciation of stool samples. For the qualitative analysis, faeces were analysed by the flotation technique using saturated sodium chloride solution and the Mac Master technique was used for the quantitative analysis as described by [4]. Risk factors were determined through the questionnaires.

\section{Statistical analysis}

Data were stored in a Microsoft Excel spread sheet and then exported to Epi Infos7 software for analysis. Summary statistics were generated using the same software. For the purpose of modeling these data, explanatory variables were first explored for associations between parasites using $\chi^{2}$ test. The prevalence of helminths parasites was compared between demographic parameters using the chi square test. They were all tested at $5 \%$ significance level.

\section{Results}

Prevalence of infection among residents

Out of the 315 stool samples examined, 21(6.7\%) were infected with at least one parasitic helminth (Table 1). Two different helminths (nematodes) were recorded. These nematodes were $A$. lumbricoides and T. trichiura, with specific prevalence of 6.7\% (21 infected) and $0.3 \%$ (1 infected) respectively.

\begin{tabular}{|l|c|c|}
\hline Parasitic status & $\begin{array}{c}\text { Number of } \\
\text { individuals }\end{array}$ & $\begin{array}{c}\text { Frequency } \\
\text { (\%) }\end{array}$ \\
\hline Infected with at least one parasite & 21 & 6.7 \\
Uninfected & 294 & 93.3 \\
\hline Total & 315 & 100.0 \\
\hline
\end{tabular}

Table 1: The overall percentage of infected and uninfected individuals.

Prevalence of parasitic helminths according to demographic parameters

Prevalence of parasitic helminths according to gender

Out of the 149 females examined, 11 (7.38\%) harbored parasites and $10(6.62 \%)$ of the 166 males were tested positive for these nematodes with no significant difference $(\mathrm{P}>0.05)$ as shown on table 2. A case of double parasitism (A. lumbricoides + T. trichiura) was observed among females.

\begin{tabular}{|l|c|c|}
\hline & \multicolumn{2}{|c|}{ Gender } \\
\hline & Female & Male \\
\hline Nematodes & N (\%) & N (\%) \\
\hline A. lumbricoides & $10(6.71)$ & $11(6.62)$ \\
\hline T. trichiura & $1(0.67)$ & - \\
\hline Total & $11(7.38)$ & $11(6.62)$ \\
\hline
\end{tabular}

Table 2: Prevalence of parasitic helminths according to gender. 
The influence of age on the prevalence of gastro-intestinal helminths

It can be noticed on table 3 that the prevalence of parasitic helminthiasis was highest in children from 6 to 10 years with an overall prevalence of $7.5 \%$. In this group, A. lumbricoides and $T$. trichiura infections had specific prevalences of $6.67 \%$ and $0.83 \%$ respectively. This was followed by those of 1 to 5 aged group with $6.30 \%$ of Ascaris infection. The least infections occurred in the 11 - 15 aged group with only 6 (7.14\%) infected with A. lumbricoides. The prevalences of $A$. lumbricoides and T. trichiura showed significant differences between the age groups $(\mathrm{P}<0.05)$.

\begin{tabular}{|l|c|c|}
\hline & \multicolumn{2}{|c|}{ Nematodes } \\
\hline Age group & A. lumbricoïdes & T. trichiura \\
\hline (Years) & N (\%) & N (\%) \\
\hline$[1-5]$ & $7(6.30)$ & - \\
\hline$[6-10]$ & $8(6.67)$ & $1(0.83)$ \\
\hline$[11-15]$ & $6(7.14)$ & - \\
\hline Total & $21(20.11)$ & $1(0.83)$ \\
\hline
\end{tabular}

Table 3: The Prevalence of helminth infections according to age groups.

The prevalence of parasitic helminths according to locality

Out of the four localities involved in this study, at least one parasitic helminth was identified in each of them except Mbouo Hospital which harbored no parasites. Ha'a showed very high prevalence of $A$. lumbricoides (8.69\%) with significant differences $(\mathrm{P}<0.05)$ between the localities (Table 4$)$.

\begin{tabular}{|l|c|c|}
\hline & \multicolumn{2}{|c|}{ Nematodes } \\
\hline Localities & Ascaris lumbricoïdes & Trichuris trichiura \\
\hline & N (\%) & N (\%) \\
\hline Kamgo & $7(7.78)$ & - \\
\hline Ha'a & $10(8.69)$ & - \\
\hline Tesse & $4(4.00)$ & $1(1.00)$ \\
\hline Mbouo Hospital & 0 & 0 \\
\hline Total & $21(20.47)$ & $1(1.00)$ \\
\hline
\end{tabular}

Table 4: The prevalence of parasitic helminths among localities.
Intensity and degree of infections among residents Intensity of infection (mean EPG) among residents

The specific intensity of infection is shown on table 5 . Out of the 315 stool samples examined, $A$. lumbricoides (6971.428614662.4228) recorded the highest intensity of infection and T. trichiura the lowest $(50 \pm 00)$.

\begin{tabular}{|l|c|c|}
\hline Nematodes & $\begin{array}{c}\text { Number of } \\
\text { Infected } \\
\text { individuals }\end{array}$ & Egg per gram (Mean \pm SD) \\
\hline Ascaris lumbricoides & 21 & $6971.4286 \pm 14662.4228$ \\
\hline Trichuris trichiura & 1 & $50 \pm 0$ \\
\hline
\end{tabular}

Table 5: Distribution according to fecal concentration of eggs.

\section{Degree of infection among residents of Bandjoun}

In general, $22.7 \%$ of infections were light infections, $13.6 \%$ moderate and $63.7 \%$ heavy infections. All T. trichiura (100\%) infections were light infections. A. lumbricoides recorded $66.7 \%$ of heavy infections; $14.3 \%$ moderate and 19\% light infections (Table $6)$.

\begin{tabular}{|c|c|c|c|c|}
\hline & \multicolumn{4}{|c|}{ Intensity of infection } \\
\hline & Light & Moderate & Heavy & Total \\
\hline Nematodes & $\mathrm{N}(\%)$ & $\mathrm{N}(\%)$ & $\mathrm{N}(\%)$ & $\mathrm{N}$ \\
\hline Ascaris lumbricoïdes & $4(19)$ & $3(14.3)$ & $14(66.7)$ & 21 \\
\hline Trichuris trichiura & $1(100)$ & & & 1 \\
\hline Total & $5(22.7)$ & $3(13.6)$ & $14(63.7)$ & 22 \\
\hline
\end{tabular}

Table 6: Degree of infection.

\section{Risk factors associated of infections}

Table 7 shows that seventeen (10.2\%) participants raised pigs around the households and were significantly more infected $(\mathrm{P}=$ $0.02<0.05$ ) than those who did not. Therefore, pig raising was significantly associated to the presence of soil-transmitted helminths in children. Not washing hands before meals is also related to the presence of soil-transmitted helminths $(\mathrm{P}=0.03<0.05)$ in children. Indeed, $9 \%$ of those who did not comply with hand hygiene rules harbored parasites compared to only $1.2 \%$ of positive cases among those who usually washed their hands. Other potential risk factors were not associated with the infection. 


\begin{tabular}{|c|c|c|c|c|c|}
\hline \multirow[b]{2}{*}{ Risk factors } & \multicolumn{5}{|c|}{ Soil-Transmitted helminths } \\
\hline & $\begin{array}{c}\text { Yes } \\
\text { N (\%) }\end{array}$ & $\begin{array}{c}\text { No } \\
\text { N (\%) }\end{array}$ & $\begin{array}{l}\text { Total } \\
\text { N (\%) }\end{array}$ & $\chi^{2}$ & P-value \\
\hline \multicolumn{6}{|c|}{ Drinking Water Supply Problems } \\
\hline Yes & $15(6.1)$ & $230(93.9)$ & $245(100)$ & 1.9 & 0.38 \\
\hline No & $6(10)$ & $54(90)$ & $60(100)$ & & \\
\hline \multicolumn{6}{|c|}{ Water treatment } \\
\hline Yes & $16(8.6)$ & $169(91.4)$ & $185(100)$ & 3.08 & 0.21 \\
\hline No & $5(4.2)$ & $115(95.8)$ & $120(100)$ & & \\
\hline \multicolumn{6}{|c|}{ Hand washing } \\
\hline Yes & $1(1.2)$ & $83(98.8)$ & $84(100)$ & 6.78 & 0.03 \\
\hline No & $20(9)$ & $201(91)$ & $221(100)$ & & \\
\hline \multicolumn{6}{|c|}{ Fruit washing } \\
\hline Yes & $2(9.5)$ & $19(90.5)$ & $21(100)$ & 0.99 & 0.61 \\
\hline No & $19(6.7)$ & $265(93.3)$ & $284(100)$ & & \\
\hline \multicolumn{6}{|c|}{ Presence of latrines } \\
\hline Yes & $21(6.9)$ & $283(93.1)$ & $304(100)$ & 081 & 0.66 \\
\hline No & $0(0)$ & $1(100)$ & $1(100)$ & & \\
\hline \multicolumn{6}{|c|}{ Presence of feces around the household } \\
\hline Yes & $8(6.3)$ & $120(93.8)$ & $128(100)$ & 0.88 & 0.64 \\
\hline No & $13(7.3)$ & $164(92.7)$ & $177(100)$ & & \\
\hline \multicolumn{6}{|c|}{ Land consumption } \\
\hline Yes & 19 (8.7) & $200(91.3)$ & $219(100)$ & 4.74 & 0.09 \\
\hline No & $2(2.3)$ & $84(97.7)$ & $86(100)$ & & \\
\hline \multicolumn{6}{|c|}{ Walking barefoot in the river } \\
\hline Yes & $18(8.3)$ & $198(91.7)$ & $216(100)$ & 3.23 & 0.19 \\
\hline No & $3(3.4)$ & $86(96.6)$ & $89(100)$ & & \\
\hline \multicolumn{6}{|l|}{ Pig raising } \\
\hline Yes & $17(10.2)$ & $150(89.8)$ & $167(100)$ & 7.17 & 0.02 \\
\hline No & $4(2.9)$ & $134(97.1)$ & $138(100)$ & & \\
\hline \multicolumn{6}{|c|}{ Knowledge of geohelminths } \\
\hline Yes & $21(7)$ & $281(93)$ & $302(100)$ & 0.96 & 0.61 \\
\hline No & $0(0)$ & $2(100)$ & $2(100)$ & & \\
\hline \multicolumn{6}{|c|}{ Consumption of Water contaminated by excreta } \\
\hline Yes & $11(8)$ & $114(91.2)$ & $125(100)$ & 1.51 & 0.21 \\
\hline No & $10(5.3)$ & $180(94.7)$ & $190(100)$ & & \\
\hline
\end{tabular}

Table 7: Capitulatory of risk factors related of infection obtain from the questionnaire. 


\section{Discussion}

The objective of this study was to evaluate the prevalence of soil-transmitted helminths on the one hand and the risk factors related to infection on the other hand among children living in Bandjoun, West region of Cameroon. The results obtained after the fecal analysis revealed a prevalence of $6.7 \%$. This result is higher than that of Munisi., et al. [6] in Tanzania with a prevalence of $0.9 \%$. In contrast, this prevalence is low compared to the results of two studies conducted in Babadjou (8.5\%) and Dschang (43.5\%), West Cameroon, respectively by Khan Payne., et al. [7] and Wabo., et al. [8]. also, it is contrary to that of Tchuem Tchuenté., et al. [5] in the Littoral, North-West, South and South-West Cameroon (32.5\%). This difference can be explained by variations in socio-economic status and the regularity of deworming campaigns.

Two nematodes were identified in our study including A. lumbricoides and T. trichiura with specific prevalences of $6.7 \%$ and $0.3 \%$. These results are weaker than those of Wabo., et al. [8] in Dschang which obtained 18\% for A. lumbricoides and 36\% for $T$. trichiura; but higher than that of Tchakounté., et al. [9] in Bazou, West Cameroon, which obtained $4.95 \%$ for A. lumbricoides and $0 \%$ for T. trichiura. The prevalence of $A$. lumbricoides infection was higher during the study. This could be explained by the fact that A. lumbricoides can survive an extended period of 10 years under conditions of humidity, heat in the external environment.

The prevalence of infections was highest in the locality of Ha'a (8.69\%) and lowest in the Tesse locality. This can be explained by the fact that a mass treatment campaign was carried out in Tesse to deworm children from 0 to 5 years old before the collection of samples. No positive case was obtained in Mbouo Hospital. The high prevalence in Haa could be due to poverty and insalubrity prevailing in this quarter. In this condition, many people do not go to the hospital for lack of money except in case of seriousness given that soil-transmitted helminth infections are in most cases asymptomatic.

Our data, like Wabo's., et al. [8], suggests that individuals in the age group [1-10] were the most infected. Indeed, Children in this age group still have very poor personal hygiene. They play together, eat together and very often do not take care of washing hands or washing fruits before eating as showed by Menan., et al [10].

Intensities of $A$. lumbricoides and T. trichiura infections was high for positive cases. This is contrary to the studies of Bethony., et al. [11], Munisi., et al. [6] and Khan Payne., et al. [12] who obtained $100 \%$ mild infection. In our study, the infection was moderate but the intensity was high. This is consistent with the study of Chan [13] who generally found that nematode infections are moderate and intensities are high in the gastrointestinal tract. The intensity was higher in A. lumbricoides (6971.4286 \pm 14662.4228 ) than T. trichiura $(50 \pm 00)$. This could be explained by the fact that A. lumbricoides produces more eggs (200,000 eggs per day) [14] than Trichuris.

Our study shows that not washing hands before eating and after leaving the toilet is an influential risk factor for the incidence of STHs infection in children compared to the others potential risks factors that were tested. Muslim., et al. [14] had found that not washing hands with clean water and soap had a significant influence on intestinal parasite infection. This is contrary to that of Tchakounté., et al. [9] who said that most of respondents washed their hands before and after each meal. The absence of hand washing points in the city of Bandjoun, the lack of hygiene and poverty of the population may be possible reasons for this factor.

In this study, the raising of pigs around the households is a significant risk factor related to geohelminths. Therefore, pigs would be considered major sources of zoonotic transmission from geohelminths to humans. This practice is known to be the main source of income for this population, where, although confinement breeding prevails, hygiene remains precarious. These personal observations would therefore explain the high contamination of the children of the breeders. In contrast, in the study of Muslim., et al. [14], individuals who have close contact with domestic animals such as dog and cat were highly predisposed to STH infections.

\section{Conclusion}

At the end of our study, where 315 stool samples and questionnaires were analyzed to evaluate the prevalence and risk factors related at infections of soil-transmitted helminths in Bandjoun, two soil-transmitted helminths were identified namely $A$. lumbricoides and T. trichiura; the infection rate was low and A. lumbricoides was the most common parasite in the study population; individuals in age groups [1-10], were the most infected; after analysis of the questionnaire, it appears that failure to wash hands before eating and after leaving the toilet and pig raising around the household are factors related to infections. 


\section{Acknowledgments}

I thank the people of Bandjoun and in particular the children who participated in the study. then, the Applied Biology and Ecology Research Unit of the Animal Biology Department of the University of Dschang for having accepted me in their laboratories.

\section{Conflict of Interest}

There is no conflict of interest regarding the publication of this article.

\section{Bibliography}

1. Nozais JP. "Maladies parasitaires et péril fécal: les maladies dues aux helminthes". Bulletin de la Societé de Pathologie Exotique 91 (1998): 1-7.

2. WHO. Eliminating soil transmitted helminthiasis as a public health problem in children: progress report 2011-2020 and strategic plan 2011-2020. World Health Organization (2012): 1-78.

3. Quihui-Cota L., et al. Intestinal parasitism schoolchildren periodically treated with albendazole in two sampling periods". International Journal of Medicine and Medical Sciences 5.2 (2013): 43-47.

4. Thienpont D., et al. "Le diagnostic des verminoses par examen coprologique". Beerse, Belgique, Janssen Research Fundation (1979): 1-187.

5. Tchuem Tchuenté L., et al. Mapping of schistosomiasis and soil-transmitted helminthiasis in the regions of Littoral, North-West, South and South-West Cameroon and recommendations for treatment". BMC Infection Disease 13.602 (2013).

6. Munisi DZ., et al. Soil-transmitted helminths infections, malnutrition and anaemia among primary school children in Same District". Muhimbili University of Health and Allied Sciences (2012). 1-16.

7. Khan Payne V., et al. "Prevalence and Intensity of Infection of Gastro-Intestinal parasites in Babadjou, West Region of Cameroon". International journal of Clinical and Experimental Medical Sciences 3.2 (2017): 14-22.

8. Wabo Poné J., et al. "Prevalence and intensity of infections of three neglected tropical diseases in patients consulted at a traditional health care centre in Dschang West Cameroon". Tropical Parasitology 2.1 (2012): 1-5.
9. Tchakounté N.B., et al. "Soil contamination Rate, Prevalence, Intensity of infection of Geohelminths and Associated Risk Factors among Residents in Bazou (West Cameroon)". Ethiopian Journal of Health Sciences 28.1 (2018): 63-72.

10. Menan EIH., et al. "Profil des helminthiases intestinales chez les enfants d'âge scolaire dans la ville d'Abidjan". Médecine d'Afrique Noire 44.7 (1997): 1-5.

11. Bethony J., et al. Soil-transmitted helminth infectons: ascariasis, trichuriasis, and hookworm". Lancet 367.9521 (2006): 1521-1532.

12. Khan Payne V., et al. "Efficacy of Single Dose of $500 \mathrm{mg}$ Mebendazole on Geohelminths amongst School Age Children in Bafoussam, Cameroon". British Microbiology Research Journal International 12.2 (2016): 1-9.

13. Chan MS. The global burden of intestinal nematode infections fifty years on". Parasitology Today 13.11 (1997): 438-443.

14. Muslim A., et al. "Prevalence, intensity and associated risk factors of soil transmitted helminth infections: A comparison between Negritos (indigenous) in inland jungle and those in resettlement at town peripheries". PLoS Neglected Tropical Disease 13.4 (2019): 1-22.

\section{Assets from publication with us}

- Prompt Acknowledgement after receiving the article

- Thorough Double blinded peer review

- Rapid Publication

- Issue of Publication Certificate

- High visibility of your Published work

Website: $\underline{\text { https://www.actascientific.com/ }}$

Submit Article: https://www.actascientific.com/submission.php Email us: editor@actascientific.com

Contact us: +919182824667 